\title{
Parametric Vibration Analysis of Pipes Conveying Fluid by Nonlinear Normal Modes and a Numerical Iterative Approach
}

\author{
Feng Liang ${ }^{1,2, *}$, Xiaodong Yang ${ }^{2, *}$, Wei Zhang ${ }^{2}$, Yingjing Qian ${ }^{2}$ \\ and Roderick V. N. Melnik ${ }^{3,4}$ \\ ${ }^{1}$ College of Mechanical Engineering, Yangzhou University, Yangzhou 225127, Jiangsu, \\ China \\ ${ }^{2}$ Beijing Key Laboratory of Nonlinear Vibrations and Strength of Mechanical \\ Engineering, College of Mechanical Engineering and Applied Electronics, Beijing \\ University of Technology, Beijing 100124, China \\ 3 The MS2Discovery Interdisciplinary Research Institute, M2NeT Laboratory, Wilfrid \\ Laurier University, 75 University Avenue West, Waterloo, ON, Canada, N2L 3C5 \\ ${ }^{4}$ BCAM-Basque Center for Applied Mathematics, E48009 Bilbao, Spain
}

Received 28 February 2018, Accepted (in revised version) 8 July 2018

\begin{abstract}
Nonlinear normal modes and a numerical iterative approach are applied to study the parametric vibrations of pipes conveying pulsating fluid as an example of gyroscopic continua. The nonlinear non-autonomous governing equations are transformed into a set of pseudo-autonomous ones by employing the harmonic balance method. The nonlinear normal modes are constructed by the invariant manifold method on the state space and a numerical iterative approach is adopted to obtain numerical solutions, in which two types of initial conditions for the modal coefficients are employed. The results show that both initial conditions can lead to fast convergence. The frequency-amplitude responses with some modal motions in phase space are obtained by the present iterative method. Quadrature phase difference and traveling waves are found in the time-domain complex modal analysis.
\end{abstract}

AMS subject classifications: 70K28, 70K75, 39B12

Key words: Gyroscopic continua, pipes conveying pulsating fluid, parametric vibration, nonlinear normal modes, iterative approach.

\section{Introduction}

The gyroscopic device is a kind of basic engineering structure with extensive applications in aerospace, navigation, petroleum and mechanical automation. The gyroscopic systems

${ }^{*}$ Corresponding author.

Email: jxdyang@163.com (X. D. Yang) 
can generally be classified into two categories: one is the translating materials, such as pipes conveying fluid and axially moving systems, the gyroscopic coupling of which is caused by the coupling between general coordinates of arbitrary transverse motion; the other is rotating bodies, the gyroscopic coupling of which is due to the coupling between two directions in the motion plane [1].

In whichever of the above gyroscopic systems, the presence of skew-symmetric gyroscopic operator in the governing equations limits analytical results but enriches dynamic behaviors dramatically, which has attracted much research attention to this field over years. Investigations of the gyroscopic systems were originated from the study of dynamics of the band saws [2]. Earlier research was confined to the analysis of natural frequencies, critical speed, and stability of the linear free vibrations [3]. With deeper understanding of the gyroscopic dynamics, the nonlinear properties of gyroscopic systems gradually became research focus, including the responses to external excitation and parametric resonance studied by perturbation method [4] and numerical method [5], and the mode interactions due to internal resonance [6]. Currently, great progress has been made towards exploration of various gyroscopic structures, involving the fluid-structure interaction systems [7,8], axially moving systems [9] and rotating bodies [10]. The threebody problems in celestial mechanics, a discrete gyroscopic system, are also concerned in nonlinear dynamic realm [11].

Natural frequencies and vibration responses of a non-gyroscopic system are often predicted by means of modal analysis, even in the case of mode interactions. However for a gyroscopic system, the modal analysis becomes complicated because the complex modes must be involved to capture the dynamics in nature $[12,13]$. In the classical real modal analysis, as we have known, if the coordinate/velocity of one arbitrary DOF is given, those of all the other DOFs can be represented as functions of the given coordinate/velocity. It implies that the coordinates/velocities of all DOFs will hold the same phase or the phase difference of $\pi$, which leads to an in-unison vibration. Whereas in a complex modal analysis, the coordinate/velocity of each DOF is the function of combination of the given coordinate and velocity. There thus exist any possible phase differences among the DOFs and an out-of-unison vibration is present. Current researches on complex modes are mostly regarding their applications in linear damped systems since the fundamental significance of modal analysis lies in the design of mechanical systems in linear regimes, such as the acquisition of natural frequencies and mode shapes of a dynamic structure [14,15]. Related studies have been extended to the rotor systems [16] and biomechanics [17]. Whereas in many cases, the nonlinear effects on these mechanical structures are often hard to be neglected, such that the subsequent concentrations have been put into the nonlinear modes. Among the related researches, the contribution of Nayfeh [18] is respected as a significant foundation in this area, wherein comprehensive nonlinear interactions, involving nonlinear mode couplings, have been explored and summarized by analytical, numerical and experimental approaches. With these developments, nonlinear complex modes are gradually adopted to extend the conventional linear complex modal theory into nonlinear fields, which shed a new light on the modal analy- 
sis of nonlinear systems [19-21].

As a typical gyroscopic continuum, the pipes conveying fluid have been broadly applied in various fields of modern industry and agriculture. By absorbing the kinetic energy of the flowing fluid, the pipe often vibrates intensely due to the fluid-structure interaction, namely self-excited vibration, which is hazardous or potentially risky for mechanical structures. In the past decades, theoretical and experimental researches towards the dynamics of pipes conveying fluid have been carried out extensively. Significant dynamic behaviors, including internal resonance, friction coupling and bifurcation and chaos, have been revealed, as reported in numerous literature such as that by Païdoussis [22], Ferràs et al. [23], Xu and Yang [24,25] and Wang [26]. Especially from the perspective of gyroscopic continua, $\mathrm{Ni}$ et al. [27] examined the free vibration of pipes conveying fluid via the differential transformation method. Öz and Boyaci [28] and Panda and Kar [29] carried out parametric vibration analysis of pipes conveying pulsating fluid by using the method of multiple scales. Actually, the fluid-conveying pipe behaves more like an axially moving system merely with the difference that the axially moving system moves axially by itself without static surrounding medium, while the fluid-conveying pipe is static with the fluid medium moving axially.

The parametric vibration is a significant dynamical phenomenon for a gyroscopic structure. Although plenty of achievements on the dynamics of gyroscopic continua have been made, there have not been comprehensive investigations regarding the parametric vibration problems from the modal point of view, to the best knowledge of authors, especially by using the nonlinear modes. In the present paper, the pipes conveying pulsating fluid are used as a representative example to explore the nonlinear parametric vibration responses of the gyroscopic continua. The nonlinear normal modes are constructed by employing the invariant manifold method proposed by Shaw and Pierre [30] and an efficient iterative approach is adopted to derive the numerical solution. The frequencydomain response analysis and the time-domain complex modal response analysis are both performed, which will provide in-depth interpretation for the nonlinear parametric vibrations of gyroscopic continua.

\section{A typical gyroscopic continuum: Pipes conveying fluid}

When the fluid flows through an elastic hollow cylinder at a certain relative velocity, the Coriolis force will be present due to the fluid-structure interaction. This is a typical reflection of the gyroscopic effect on the translating materials. In the following analysis, we will take the pipes conveying pulsating fluid as an example to investigate the nonlinear parametric vibration responses of a gyroscopic system. The mechanical model of a pinned-pinned uniform pipe conveying fluid is shown in Fig. 1. The pipe is assumed to be subject to transverse planar motions: $y(x, t)$ and its axis in the undeformed state coincides with the $x$-axis. We use t to denote time variable and $U$ the flow velocity. Taking the geometrical nonlinearity into account, the partial differential equation governing 


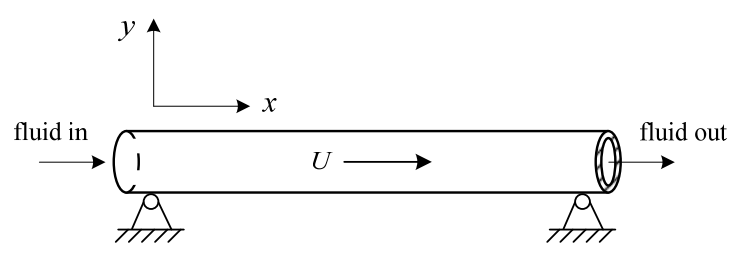

Figure 1: Mechanical model of a pinned-pinned pipe conveying fluid.

transverse vibration for such a conservative gyroscopic system can be written as [31]

$$
\begin{gathered}
E I \frac{\partial^{4} y}{\partial x^{4}}+M\left[U^{2}+\frac{\partial U}{\partial t}(L-x)\right] \frac{\partial^{2} y}{\partial x^{2}}+(M+m) \frac{\partial^{2} y}{\partial t^{2}}+2 M U \frac{\partial^{2} y}{\partial x \partial t} \\
-\left[\frac{E A_{p}}{2 L} \int_{0}^{L}\left(\frac{\partial y}{\partial x}\right)^{2} d x\right] \frac{\partial^{2} y}{\partial x^{2}}=0,
\end{gathered}
$$

where $E, I, L, A_{p}$ and $m$ are the Young's modulus, inertia moment of cross section, length, cross-sectional area and mass per unit length of the pipe, respectively, and $M$ represents the mass per unit length of the fluid conveyed.

We introduce the following dimensionless variables and parameters

$$
\begin{array}{lll}
\eta=\frac{y}{L}, & \xi=\frac{x}{L}, & \tau=\left(\frac{E I}{M+m}\right)^{\frac{1}{2}} \frac{t}{L^{2}}, \\
M_{r}=\left(\frac{M}{M+m}\right)^{\frac{1}{2}}, & u=\left(\frac{M}{E I}\right)^{\frac{1}{2}} L U, & \gamma=\frac{A_{p} L^{2}}{2 I},
\end{array}
$$

and the dimensionless harmonically fluctuated flow

$$
u=u_{0}[1+\mu \cos (2 \omega \tau)]
$$

where $u_{0}$ is the mean flow velocity, $\mu$ the amplitude of the harmonic fluctuation (assumed small) and $2 \omega$ its frequency. Substitutions of Eqs. (2.2) and (2.4) into Eq. (2.1) yield the dimensionless governing equation as follows

$$
\begin{aligned}
& \frac{\partial^{4} \eta}{\partial \xi^{4}}+u_{0}^{2} \frac{\partial^{2} \eta}{\partial \xi^{2}}+\frac{\partial^{2} \eta}{\partial \tau^{2}}+2 M_{r} u_{0} \frac{\partial^{2} \eta}{\partial \xi \partial \tau} \\
&=\mu\left[2 M_{r} u_{0} \omega(1-\xi) \sin (2 \omega \tau) \frac{\partial^{2} \eta}{\partial \xi^{2}}-2 \cos (2 \omega \tau)\left(u_{0}^{2} \frac{\partial^{2} \eta}{\partial \xi^{2}}+M_{r} u_{0} \frac{\partial^{2} \eta}{\partial \xi \partial \tau}\right)\right] \\
&+\left[\gamma \int_{0}^{1}\left(\frac{\partial \eta}{\partial \xi}\right)^{2} d \xi\right] \frac{\partial^{2} \eta}{\partial \xi^{2}} .
\end{aligned}
$$

Note that in Eq. (2.5) the higher-order terms of $\mu$ have been omitted. 
In order to introduce the nonlinear normal modes, the continuous model governed by the partial differential equation will first be discretized via the Galerkin technique with 2-term truncation

$$
\eta(\xi, \tau)=\sum_{i=1}^{2} \psi_{i}(\xi) q_{i}(\tau),
$$

where $\psi_{i}(\varepsilon)$ and $q_{i}(\tau)$ stand for the $i$ th admissible mode function and general coordinate, respectively. It is noted that some theoretical and experimental research works have pointed out that a 2-term Galerkin truncation for the supported pipes conveying fluid could attain adequate precision, and the experimental results were at least in good qualitative agreement with the theoretical ones [32,33]. Also, the results obtained later will prove that the numerical solutions can convergence fast and the accuracy of the solutions is not affected by this truncation. Now, we choose the mode functions of static pinned-pinned beams as admissible functions

$$
\psi_{r}(\xi)=\sqrt{2} \sin (r \pi \xi), \quad r=1,2,
$$

for simplicity. Substituting Eqs. (2.6) and (2.7) into Eq. (2.5) and employing the orthogonality property of the admissible mode functions, one can get a set of two-dimensional second-order ordinary differential equations

$$
\left\{\begin{array}{l}
\ddot{q}_{1}-\beta[1+\mu \cos (2 \omega \tau)] \dot{q}_{2}+\left[k_{1}+\frac{3}{16} \pi^{2} \mu \beta \omega \sin (2 \omega \tau)-2 \pi^{2} \mu u_{0}^{2} \cos (2 \omega \tau)\right] q_{1} \\
+\frac{8}{3} \mu \beta \omega \sin (2 \omega \tau) q_{2}+n_{11} q_{1}^{3}+n_{12} q_{1} q_{2}^{2}=0, \\
\ddot{q}_{2}+\beta[1+\mu \cos (2 \omega \tau)] \dot{q}_{1}+\left[k_{2}+\frac{3}{4} \pi^{2} \mu \beta \omega \sin (2 \omega \tau)-8 \pi^{2} \mu u_{0}^{2} \cos (2 \omega \tau)\right] q_{2} \\
\quad+\frac{2}{3} \mu \beta \omega \sin (2 \omega \tau) q_{1}+n_{21} q_{2} q_{1}^{2}+n_{22} q_{2}^{3}=0,
\end{array}\right.
$$

where

$$
\begin{array}{ll}
\beta=\frac{16}{3} M_{r} u_{0}, \quad k_{1}=-\pi^{2} u_{0}^{2}+\pi^{4}, & k_{2}=-4 \pi^{2} u_{0}^{2}+16 \pi^{4}, \\
n_{11}=\pi^{4} \gamma, \quad n_{12}=4 \pi^{4} \gamma, \quad n_{21}=n_{12}, & n_{22}=16 \pi^{4} \gamma .
\end{array}
$$

\section{Construction of the nonlinear normal modes}

In this section, the nonlinear normal modes of the present gyroscopic system will be constructed with the invariant manifold method, by which the parametric resonances of the gyroscopic system are discussed. It should be mentioned that some general solution approaches have been developed to deal with the continuous systems, inclusive 
of gyroscopic systems, with cubic nonlinearity, especially the method of multiple scales employed by Özhan and Pakdemirli [34] and Ghayesh et al. [35], by which closed-form solutions can be obtained to clearly reflect the dynamical evolutions of the system with parameters. However, these analytical solutions are valid only within a limited range of parameters. In order to find accurate solutions in an extensive region, numerical procedures may have to be applied. In the following analysis, the nonlinear normal modes in conjunction with a numerical iterative approach proposed by Avramov [36] are used to investigate the responses to the parametric resonances of the gyroscopic system. To efficiently perform the iterative loop, we should first select an appropriate initial value. Herein two types of initial conditions for the modal coefficients are considered. The first case takes the conditions of the corresponding autonomous part of the parametric excited system and the second case utilizes the decoupled coordinate of the non-autonomous system as initial conditions. The results obtained by different initial iterative conditions can be compared and verified mutually.

For the first initial conditions, let

$$
\left[p_{1}, p_{2}\right]=\left[\dot{q}_{1}, \dot{q}_{2}\right],
$$

and $\mu$ is assumed to be zero, Eq. (2.8) is then transformed into a set of first-order state equations governing the nonlinear free vibration of the pipe conveying fluid

$$
\left.\begin{array}{ll}
\dot{q}_{1}=p_{1}, & \dot{p}_{1}=f_{1}\left(q_{1}, q_{2} ; p_{1}, p_{2}\right)=\beta p_{2}-k_{1} q_{1}-n_{11} q_{1}^{3}-n_{12} q_{1} q_{2}^{2} \\
\dot{q}_{2}=p_{2}, & \dot{p}_{2}=f_{2}\left(q_{1}, q_{2} ; p_{1}, p_{2}\right)=-\beta p_{1}-k_{2} q_{2}-n_{21} q_{2} q_{1}^{2}-n_{22} q_{2}^{3}
\end{array}\right\} .
$$

The invariant manifold method is now implemented to obtain the initial modal coefficients of the coupled autonomous system. Considering the gyroscopic coupling of the system and only the cubic nonlinearity of Eq. (3.2), we can assume a purely modal motion by requiring that $q_{1}, p_{1}, q_{2}$ and $p_{2}$ are related nonlinearly as

$$
\begin{aligned}
& q_{1}=w, \quad p_{1}=v, \\
& q_{2}=a_{1} w+a_{2} v+a_{3} w^{3}+a_{4} w^{2} v+a_{5} w v^{2}+a_{6} v^{3}, \\
& p_{2}=b_{1} w+b_{2} v+b_{3} w^{3}+b_{4} w^{2} v+b_{5} w v^{2}+b_{6} v^{3} .
\end{aligned}
$$

Substituting Eqs. (3.2) and (3.3) into the time derivatives of the last two equations of Eq. (3.3), and collecting the coefficients of $w, v, w^{3}, w^{2} v, w v^{2}$ and $v^{3}$ the nonlinear equations with respect to $a_{j}$ and $b_{j}, j=1 \sim 6$ can be yielded, which have been given in Appendix. The first four of these equations can be solved for the linear modal coefficients as

$$
\begin{aligned}
& a_{1}=0, \quad a_{2}=\frac{-\left(\beta^{2}+k_{1}-k_{2}\right) \mp \sqrt{\left(\beta^{2}+k_{1}-k_{2}\right)^{2}+4 \beta^{2} k_{2}}}{2 \beta k_{2}}, \\
& b_{1}=\frac{-\beta^{2}+k_{1}-k_{2} \pm \sqrt{\left(\beta^{2}+k_{1}-k_{2}\right)^{2}+4 \beta^{2} k_{2}}}{2 \beta}, \quad b_{2}=0 .
\end{aligned}
$$


Along with these linear modal coefficients, the following nonlinear modal coefficients can be obtained analytically

$$
\begin{aligned}
& a_{3}=a_{5}=b_{4}=b_{6}=0, \\
& a_{6}=\left(s_{4}-2 s_{1} s_{2}\right) /\left(2 s_{1}^{2}-s_{3}\right), \quad a_{4}=s_{1} a_{6}+s_{2}, \\
& b_{3}=\left(\beta a_{4} b_{1}-n_{11} a_{2}-k_{1} a_{4}\right) /\left(1-\beta a_{2}\right), \quad b_{5}=-k_{2} a_{6}-n_{22} a_{2}^{3},
\end{aligned}
$$

where the expressions of $s_{1} \sim s_{4}$ are given in Appendix. Thus, the initial values by the corresponding autonomous system are determined. Now we treat the non-autonomous equations (2.8). Substituting the last two equations of Eq. (3.3) into the first equation of Eq. (2.8), we can obtain a decoupled differential equation with respect to $q_{1}$

$$
\begin{aligned}
& \ddot{q}_{1}-\beta[1+\mu \cos (2 \omega \tau)] \dot{q}_{2}\left(q_{1}, \dot{q}_{1}\right)+\left[k_{1}+\frac{3}{16} \pi^{2} \mu \beta \omega \sin (2 \omega \tau)-2 \pi^{2} \mu u_{0}^{2} \cos (2 \omega \tau)\right] q_{1} \\
& +\frac{8}{3} \mu \beta \omega \sin (2 \omega \tau) q_{2}\left(q_{1}, \dot{q}_{1}\right)+n_{11} q_{1}^{3}+n_{12} q_{1} q_{2}^{2}\left(q_{1}, \dot{q}_{1}\right)=0 .
\end{aligned}
$$

Assume that the system (3.6) holds the following periodic motion

$$
q_{1}=A_{1} \cos (\omega \tau)+B_{1} \sin (\omega \tau),
$$

where $A_{1}$ and $B_{1}$ can be determined by the harmonic balance method. At the same time, according to Eq. (3.7), the following expressions can be derived

$$
\begin{aligned}
& \cos (2 \omega \tau)=\alpha_{1} q_{1}^{2}+\alpha_{2} \dot{q}_{1}^{2}+\alpha_{3} q_{1} \dot{q}_{1}, \\
& \sin (2 \omega \tau)=\frac{\omega \alpha_{3}}{2} q_{1}^{2}-\frac{\alpha_{3}}{2 \omega} \dot{q}_{1}^{2}+2 \omega \alpha_{2} q_{1} \dot{q}_{1},
\end{aligned}
$$

where

$$
\alpha_{1}=\frac{A_{1}^{2}-B_{1}^{2}}{\left(A_{1}^{2}+B_{1}^{2}\right)^{2}}, \quad \alpha_{2}=\frac{B_{1}^{2}-A_{1}^{2}}{\omega^{2}\left(A_{1}^{2}+B_{1}^{2}\right)^{2}}, \quad \alpha_{3}=\frac{4 A_{1} B_{1}}{\omega\left(A_{1}^{2}+B_{1}^{2}\right)^{2}} .
$$

Substitute Eq. (3.8) into Eq. (2.8) and a pseudo-autonomous gyroscopic system will be derived, which still holds a cubic nonlinearity form. Therefore, the nonlinear normal modes of the gyroscopic system with parametric excitation can also be taken as the same form of Eq. (3.3) with the coefficients to be determined. Repeat the steps from Eq. (3.1) to Eq. (3.5) and the modal coefficients for the first iteration can be obtained.

For the second initial conditions, we consider the case of the first-mode parametric resonance, at which the amplitude of the modal motion of $q_{1}$ is much larger than that of $q_{2}$. Such motions can be approximately described by taking $q_{2}=0$ and the system (2.8) thus has the following form

$$
\ddot{q}_{1}+\left[k_{1}+\frac{3}{16} \pi^{2} \mu \beta \omega \sin (2 \omega \tau)-2 \pi^{2} \mu u_{0}^{2} \cos (2 \omega \tau)\right] q_{1}+n_{11} q_{1}^{3}=0 .
$$

Repeat the steps starting from Eq. (3.7) and the first iteration for this initial conditions can be completed. 


\section{Results and discussion}

In this section, the fundamental (first-mode) parametric resonance will be analyzed by numerical examples. We firstly inspect the convergence of the present iterative loop, and meanwhile compare the iterative results of two initial conditions. In the following examples, the parameter values that may be used are all taken as: $u_{0}=2, M r=0.8, \omega=7.41$, $\mu=0.3, \gamma=0.03$. Table 1 and Table 2 list the iterative results of the modal coefficients with the initial conditions of the coupled autonomous system and decoupled non-autonomous system, respectively. It can be seen that there already has a higher precision after the third iteration for both initial conditions. Moreover, the final calculation results of the modal coefficients with the two initial conditions achieve a good agreement. However, it should be noted that the initial conditions of decoupled non-autonomous system are of significance only in the neighbourhood of resonance points, while the initial conditions of coupled autonomous system can be employed anywhere.

Table 1: The iterative results of the modal coefficients with the initial conditions of coupled autonomous system.

\begin{tabular}{||c|cccc||}
\hline Modal coefficients & \multicolumn{4}{|c||}{ The $n$-th iteration } \\
\hline & 1st & 2nd & 3rd & 4th \\
\hline$A_{1}$ & -2.775 & -2.604 & -2.584 & -2.581 \\
\hline$B_{1}$ & 1.520 & 1.521 & 1.529 & 1.530 \\
\hline$a_{3} / E-04$ & 5.436 & 6.335 & 6.458 & 6.473 \\
\hline$a_{4} / E-05$ & -0.512 & -1.094 & -1.183 & -1.195 \\
\hline$a_{5} / E-05$ & 7.213 & 8.292 & 8.432 & 8.448 \\
\hline$a_{6} / E-06$ & 1.526 & 1.428 & 1.390 & 1.383 \\
\hline$b_{3} / E-02$ & -0.789 & -1.008 & -1.025 & -1.026 \\
\hline$b_{4} / E-03$ & -5.519 & -5.913 & -5.931 & -5.930 \\
\hline$b_{5} / E-04$ & -3.964 & -4.362 & -4.394 & -4.396 \\
\hline$b_{6} / E-05$ & 7.138 & 8.203 & 8.340 & 8.357 \\
\hline
\end{tabular}

Table 2: The iterative results of the modal coefficients with the initial conditions of decoupled non-autonomous system.

\begin{tabular}{||c|cccc||}
\hline Modal coefficients & \multicolumn{4}{|c||}{ The $n$-th iteration } \\
\hline & 1st & 2nd & 3rd & 4th \\
\hline$A_{1}$ & -2.545 & -2.578 & -2.580 & -2.581 \\
\hline$B_{1}$ & 1.353 & 1.516 & 1.530 & 1.531 \\
\hline$a_{3} / E-04$ & 6.376 & 6.475 & 6.478 & 6.475 \\
\hline$a_{4} / E-05$ & -0.900 & -1.179 & -1.197 & -1.197 \\
\hline$a_{5} / E-05$ & 8.514 & 8.465 & 8.455 & 8.451 \\
\hline$a_{6} / E-06$ & 1.967 & 1.427 & 1.385 & 1.382 \\
\hline$b_{3} / E-02$ & -1.299 & -1.049 & -1.028 & -1.026 \\
\hline$b_{4} / E-03$ & -6.726 & -5.995 & -5.936 & -5.930 \\
\hline$b_{5} / E-04$ & -4.894 & -4.438 & -4.400 & -4.396 \\
\hline$b_{6} / E-05$ & 8.427 & 8.374 & 8.363 & 8.360 \\
\hline
\end{tabular}




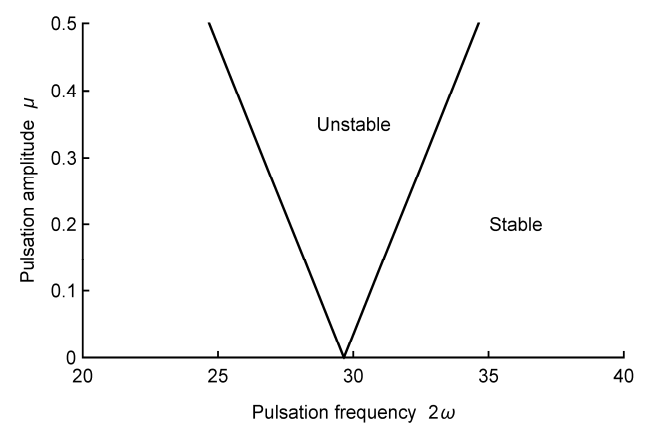

Figure 2: The stability region of the system considered.

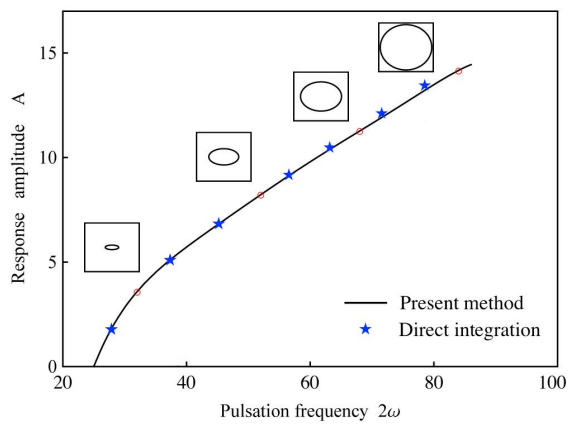

(a) The first general coordinate $q_{1}$

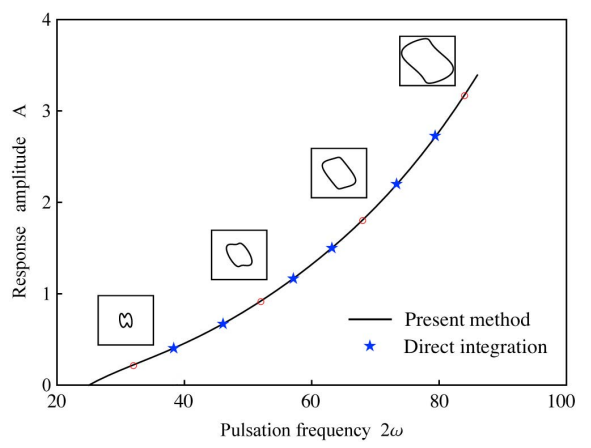

(b) The second general coordinate $q_{2}$

Figure 3: The frequency-amplitude responses and modal motions in phase space.

Next, we carry out a frequency-domain analysis for the present parameter-excited gyroscopic system. Before discussing the response characteristics, a stability inspection should be conducted via the linear system. By using the parameter values given in the foregoing, the stability region in the $\omega-\mu$ plane is plotted in Fig. 2 by the averaging method, which is a dependable perturbation approach employed in much literature [31, 37,38]. Note that areas inside and outside the boundary in the figure indicate unstable (parametric resonance) and stable regions, respectively. It is found that a linear stability boundary is present in Fig. 2, because the damping is neglected. Moreover, the area of unstable region will be increased, that is, the stability of the system will be impaired, with increase of the excitation amplitude.

Fig. 3 depicts the nonlinear frequency-amplitude responses of the two general coordinates, $q_{1}$ and $q_{2}$, calculated by the present iterative approach. Selected modal motions of the general coordinates on state space are also presented in the figure. To further verify the obtained results, the numerical responses by direct integration of Eq. (2.8) are plotted in Fig. 2 with blue asterisks. A good agreement can be found between the two results, which demonstrates the effectiveness of the current invariant manifold method and iter- 


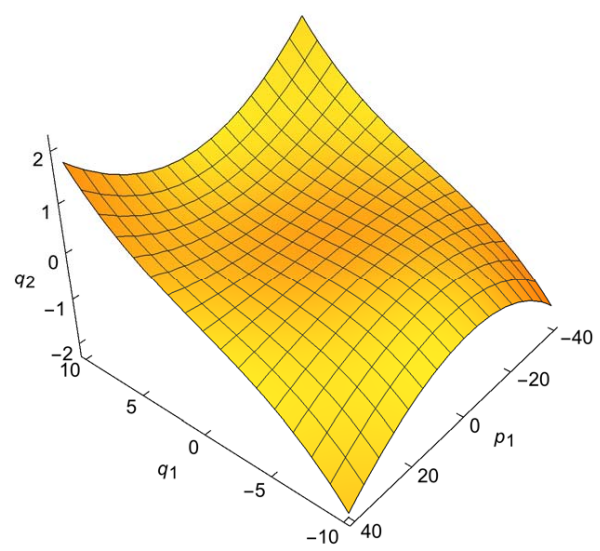

(a) $q_{2}$ as function of $q_{1}$ and $p_{1}$

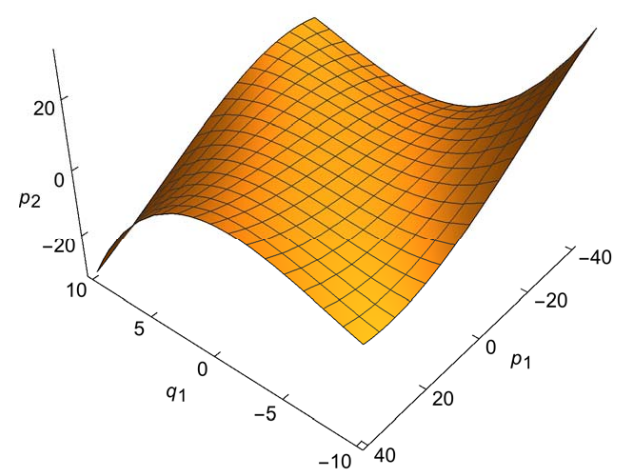

(b) $p_{2}$ as function of $q_{1}$ and $p_{1}$

Figure 4: The nonlinear invariant manifolds for the parameter-excited gyroscopic system.

ative approach. Moreover, although both of $q_{1}$ and $q_{2}$ show a hardening response characteristic, as displayed in Fig. 3(a) and Fig. 3(b), there is still evident distinction between them. The reason is that in the present nonlinear modal analysis, according to Eqs. (3.3) and (3.7), $q_{1}$ and $p_{1}$ perform a simple harmonic motion, while $q_{2}$ is the complicated nonlinear function of $q_{1}$ and its velocity $p_{1}$.

The nonlinear invariant manifolds of the system are plotted in Fig. 4. We can observe in the figure that the manifolds are not planar owing to the effect of nonlinearity. The nonlinear invariant manifold of $q_{2}$ is a warping surface and that of $p_{2}$ is a waved one. Furthermore, from the physical point of view, the "invariance" means that any motion starting from the manifold will remain in it for all the time, and the system behaves as a nonlinear single DOF system on the manifold. For the present parameter-excited gyroscopic system, periodic orbits of the modal motions can be obtained for different amplitudes in phase space, as shown in Fig. 3, and the nonlinear invariant manifolds are actually constructed by all the possible three-dimensional periodic orbits with respect to $q_{2}, q_{1}, p_{1}$ or $p_{2}, q_{1}, p_{1}$.

Now we investigate the time-domain responses of the system by taking advantage of nonlinear complex modes. In terms of Eq. (3.7), we can describe the period modal motion of $q_{1}$ in complex form

$$
q_{1}=A e^{i \omega \tau}
$$

Substituting Eq. (4.1) into Eq. (3.3), $p_{1}, q_{2}$ and $p_{2}$ can also be expressed in complex form. In order to examine the phase relations of the two DOFs, we plot the time histories of $q_{1}$, $p_{1}, q_{2}$ and $p_{2}$ during parametric vibrations in Fig. 5. In this figure and the following Fig. 6, the parameter values used are $A=1, \omega=17$. It is found a $\pi / 2$ phase difference exists between $q_{1}$ and $q_{2}$, which is a typical phenomenon of the gyroscopic system. In general, the coordinate/velocity of the second DOF is mainly associated with the velocity/coordinate 


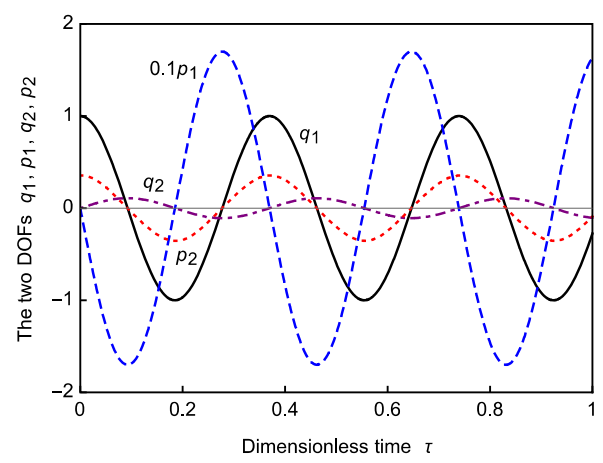

Figure 5: The time histories of $q_{1}, p_{1}, q_{2}$ and $p_{2}$ during parametric vibrations.

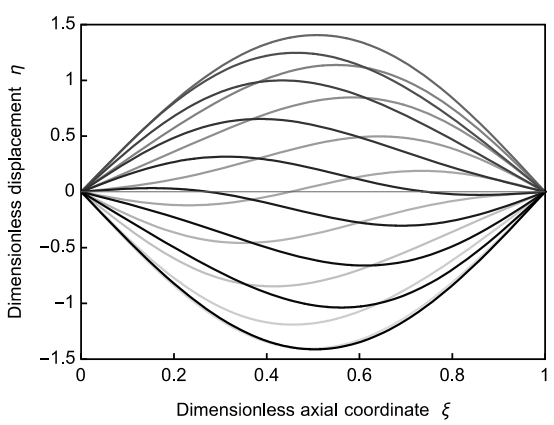

(a) A pipe conveying pulsating fluid (the gyroscopic system)

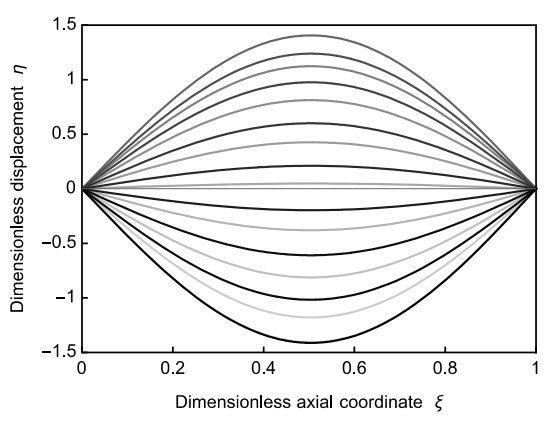

(b) A pipe filled with static liquid (the non-gyroscopic system)

Figure 6: The snapshots during modal motions.

of the first DOF, which will result in an out-of-unison vibration of the present gyroscopic system.

To clearly exhibit the complex gyroscopic mode phenomenon, by substituting the general coordinates and velocities $q_{1}, p_{1}, q_{2}$ and $p_{2}$ into Eq. (2.6), we can obtain the vibrations of the pipes on the physical coordinates. In Fig. 6(a) the snapshot of the parametric vibrations during one period process is presented for the pipes conveying fluid. For better comparison, that of the vibration process for the pipe without moving fluid, which is a non-gyroscopic system, is also plotted in Fig. 6(b). In both figures, the curve colours from shallow to deep indicate the direction of time going. It can be observed that unlike a vibration with fixed nodes in non-gyroscopic systems, which shows the "standing waves", the nodes of the gyroscopic system are shifting with time, which shows the "traveling waves". It is also found that the wave for the first mode is travelling leftward for the present modal motion whereas the fluid flows rightward. This is a significant distinction of vibrations between gyroscopic and non-gyroscopic structures. 


\section{Conclusions}

This paper investigates the parametric vibrations of gyroscopic continua from a modal perspective. The pipes conveying pulsating fluid are used as a typical example of gyroscopic structures. The nonlinear normal modes for such gyroscopic system are constructed by the invariant manifold method. An iterative approach is applied to obtain numerical solutions and the frequency-domain and time-domain response analysis are carried out to show the vibrations due to parametric resonances. The fast convergence of the response solutions proves the high efficiency of the present iterative approach. The amplitude responses to the pulsating frequencies are presented by the proposed numerical method. The quadrature phase difference and traveling waves are found in the time-domain complex modal analysis of the pipes conveying fluid.

Moreover, it should be noted that the present solution method is in nature a semianalytical and semi-numerical method, which consists of two procedures: nonlinear normal modes and numerical iteration. It is applicable for any dynamical system with finite degrees of freedom, including high nonlinear systems, in which just more terms will be involved. The solution precision mainly depends on the iteration times, and has nothing to do with the nonlinearity.

\section{Appendix}

Substituting Eqs. (3.2) and (3.3) into the time derivatives of the last two equations of Eq. (3.3), and collecting the coefficients of $w, v, w^{3}, w^{2} v, w v^{2}$ and $v^{3}$, the nonlinear equations with respect to $a_{j}$ and $b_{j}, j=1 \sim 6$ can be yielded as

$$
\begin{array}{ll}
w \text { term: } \quad & b_{1}-\beta a_{2} b_{1}+k_{1} a_{2}=0, \\
& \beta b_{1} b_{2}-k_{1} b_{2}+k_{2} a_{1}=0, \\
& a_{1}-b_{2}+\beta a_{2} b_{2}=0, \\
& b_{1}+\beta b_{2}^{2}+k_{2} a_{2}+\beta=0, \\
& b_{3}+n_{11} a_{2}+n_{12} a_{1}^{2} a_{2}-\beta a_{4} b_{1}-\beta a_{2} b_{3}+k_{1} a_{4}=0, \\
& n_{11} b_{2}+n_{12} a_{1}^{2} b_{2}-n_{21} a_{1}-n_{22} a_{1}^{3}-\beta b_{2} b_{3}-\beta b_{1} b_{4}+k_{1} b_{4}-k_{2} a_{3}=0, \\
w^{2} v \text { term }: \quad & 3 a_{3}-b_{4}-2 n_{12} a_{1} a_{2}^{2}+2 \beta a_{5} b_{1}+\beta a_{4} b_{2}+\beta a_{2} b_{4}-2 k_{1} a_{5}=0, \\
& 3 b_{3}-2 n_{12} a_{1} a_{2} b_{2}+n_{21} a_{2}+3 n_{22} a_{1}^{2} a_{2}+2 \beta b_{2} b_{4}+2 \beta b_{1} b_{5}-2 k_{1} b_{5}+k_{2} a_{4}=0, \\
w v^{2} \text { term: } \quad & 2 a_{4}-b_{5}-n_{12} a_{2}^{3}+3 \beta a_{6} b_{1}+2 \beta a_{5} b_{2}+\beta a_{2} b_{5}-3 k_{1} a_{6}=0, \\
& 2 b_{4}-n_{12} a_{2}^{2} b_{2}+3 n_{22} a_{1} a_{2}^{2}+3 \beta b_{2} b_{5}+3 \beta b_{1} b_{6}-3 k_{1} b_{6}+k_{2} a_{5}=0, \\
v^{3} \text { term: } \quad & a_{5}-b_{6}+3 \beta a_{6} b_{2}+\beta a_{2} b_{6}=0, \\
& b_{5}+n_{22} a_{2}^{3}+4 \beta b_{2} b_{6}+k_{2} a_{6}=0 .
\end{array}
$$


The expressions of $s_{1} \sim s_{4}$ in Eq. (3.5) are given as follows

$$
\begin{aligned}
& s_{1}=\frac{1}{2}\left(\beta k_{2} a_{2}-k_{2}-3 \beta b_{1}+3 k_{1}\right), \\
& s_{2}=\frac{1}{2}\left(\beta n_{22} a_{2}^{4}-n_{22} a_{2}^{3}+n_{12} a_{2}^{3}\right), \\
& s_{3}=2\left(\beta^{2} k_{2} a_{2} b_{1}-\beta k_{1} k_{2} a_{2}-\beta k_{2} b_{1}+k_{1} k_{2}\right), \\
& s_{4}=2\left(\beta^{2} n_{22} a_{2}^{4} b_{1}-\beta k_{1} n_{22} a_{2}^{4}-\beta n_{22} a_{2}^{3} b_{1}+k_{1} n_{22} a_{2}^{3}\right)-\beta n_{21} a_{2}^{2}+n_{21} a_{2}-3 n_{11} a_{2} .
\end{aligned}
$$

\section{Acknowledgements}

This study was partially funded by the National Natural Science Foundation of China (Grant Nos. 11672189, 11672007), the postdoctoral fund of Beijing Chaoyang District (Grant No. Q5001015201602) and the Program Funded by Liaoning Province Education Administration (Grant No. L2016010). Prof. X.-D. Yang was founded by the Key Laboratory of Vibration and Control of Aero-Propulsion System Ministry of Education, Northeastern University (VCAME201601). Prof. Melnik was funded by the Natural Sciences and Engineering Research Council (NSERC) of Canada, the Canada Research Chair (CRC) program, and the Bizkaia Talent Grant under the Basque Government through the BERC 2014-2017 program, as well as Spanish Ministry of Economy and Competitiveness MINECO: BCAM Severo Ochoa excellence accreditation SEV-2013-0323.

\section{References}

[1] S. A. A. HOSSEINI AND S. E. KHADEM, Free vibrations analysis of a rotating shaft with nonlinearities in curvature and inertia, Mech. Mach. Theory, 44 (2009), pp. 272-288.

[2] JR C. D. MOTE, A study of band saw vibrations, J. Franklin I., 279 (1965), pp. 430-444.

[3] J. A. WICKERT AND C. D. MOTE, Classical vibration analysis of axially moving continua, AMSE J. Appl. Mech., 57 (1990), pp. 738-744.

[4] R. A. MALOOKANI AND W. T. VAN HORSSEN, On resonances and the applicability of Galerkin's truncation method for an axially moving string with time-varying velocity, J. Sound Vib., 344 (2015), pp. 1-17.

[5] X. D. YANG, L. Q. CHEN AND J. W. ZU, Vibrations and stability of an axially moving rectangular composite plate, AMSE J. Appl. Mech., 78 (2011), 011018.

[6] M. H. GHAYESH, S. KAZEMIRAD AND M. AMABILI, Coupled longitudinal-transverse dynamics of an axially moving beam with an internal resonance, Mech. Mach. Theory, 52 (2012), pp. 18-34.

[7] E. Tubaldi, M. AmABiLi AND M. P. PÄ̈DOussis, Fluid-structure interaction for nonlinear response of shells conveying pulsatile flow, J. Sound Vib., 371 (2016), pp. 252-276.

[8] B. R. BinUlal, A. RAJAN, M. UNNIKRISHNAN AND J. KOCHUPILlai, Experimental determination of time lag due to phase shift on a flexible pipe conveying fluid, Measurement, 83 (2016), pp. 86-95.

[9] K. MARYNOWSKI AND T. KAPITANIAK, Dynamics of axially moving continua, Int. J. Mech. Sci., 81 (2014), pp. 26-41. 
[10] J. R. BANERJEE AND D. KENNEDY, Dynamic stiffness method for inplane free vibration of rotating beams including Coriolis effects, J. Sound Vib., 333 (2014), pp. 7299-7312.

[11] R. L. ANDERSON, S. CAMPAGNOLA AND G. LANTOINE, Broad search for unstable resonant orbits in the planar circular restricted three-body problem, Celest. Mech. Dyn. Astr., 124 (2016), pp. 177-199.

[12] J. A. WICKERT, Transient vibration of gyroscopic systems with unsteady superposed motion, J. Sound Vib., 195 (1996), pp. 797-807.

[13] X. D. YANG, S. YANG, Y. J. QIAN, W. ZHANG AND R. V. N. MELNIK, Modal analysis of the gyroscopic continua: comparison of continuous and discretized models, AMSE J. Appl. Mech., 83 (2016), 084502.

[14] N. Alati, G. FAilla AND A. SAntini, Complex modal analysis of rods with viscous damping devices, J. Sound Vib., 333 (2014), pp. 2130-2163.

[15] X. XING AND B. F. FEENY, Complex modal analysis of a nonmodally damped continuous beam, ASME J. Vib. Acoust., 137 (2015), 041006.

[16] D. J. HAN, Complex harmonic modal analysis of rotor systems, J. Mech. Sci. Technol., 29 (2015), pp. 2735-2746.

[17] B. F. FEENY AND A. K. FEENY, Complex modal analysis of the swimming motion of a whiting, ASME J. Vib. Acoust., 135 (2013), 021004.

[18] A. H. NAYFEH, Nonlinear Interactions: Analytical, Computational and Experimental Methods, Wiley-Interscience, New York, 2000.

[19] J. J. SinOU, F. THOUVEREZ AND L. JEZEQUEL, Stability analysis and non-linear behaviour of structural systems using the complex non-linear modal analysis (CNLMA), Comput. Struct., 84 (2006), pp. 1891-1905.

[20] H. Boudaoud, S. Belouettar, E. M. DAyA AND M. P. Ferry, A numerical method for nonlinear complex modes with application to active-passive damped sandwich structures, Eng. Struct., 31 (2009), pp. 284-291.

[21] S. G. DOU AND J. S. JENSEN, Optimization of hardening/softening behavior of plane frame structures using nonlinear normal modes, Comput. Struct., 164 (2016), pp. 63-74.

[22] M. P. PAÏDOUSSIS, Fluid-Structure Interactions: Slender Structures and Axial Flow, Second ed., Academic Press, London, 2014.

[23] D. Ferràs, P. A. MANso, A. J. Schleiss AND D. I. C. Covas, Fluid-structure interaction in straight pipelines: Friction coupling mechanisms, Comput. Struct., 175 (2016), pp. 74-90.

[24] J. XU AND Q. B. YANG, Flow-induced internal resonances and mode exchange in horizontal cantilevered pipe conveying fluid (I), Appl. Math. Mech. Eng., 27 (2006), pp. 935-941.

[25] J. XU AND Q. B. YANG, Flow-induced internal resonances and mode exchange in horizontal cantilevered pipe conveying fluid (II), Appl. Math. Mech. Eng., 27 (2006), pp. 943-951.

[26] L. WANG AND Q. NI, In-plane vibration analyses of curved pipes conveying fluid using the generalized differential quadrature rule, Comput. Struct., 86 (2008), pp. 133-139.

[27] Q. Ni, Z. L. ZHANG AND L. WANG, Application of the differential transformation method to vibration analysis of pipes conveying fluid, Appl. Math. Comput., 217 (2011), pp. 7028-7038.

[28] H. R. ÖZ AND H. BOYACI, Transverse vibrations of tensioned pipes conveying fluid with timedependent velocity, J. Sound Vib., 236 (2000), pp. 259-276.

[29] L. N. PANDA AND R. C. KAR, Nonlinear dynamics of a pipe conveying pulsating fluid with the combination, principal parametric and internal resonances, J. Sound Vib., 309 (2008), pp. 375-406.

[30] S. W. SHAW AND C. PIERRE, Normal modes for non-linear vibratory systems, J. Sound Vib., 164 (1993), pp. 85-124.

[31] J. D. JIN AND Z. Y. SONG, Parametric resonances of supported pipes conveying pulsating fluid, J. 
Fluid Struct., 20 (2005), pp. 762-783.

[32] M. P. PAÏDOUSSIS AND N. T. IssID, Dynamic stability of pipes conveying fluid, J. Sound Vib., 33 (1974), pp. 267-294.

[33] M. P. PAÏDOUSSIS AND N. T. ISSID, Experiments on parametric resonance of pipes containing pulsatile flow, J. Appl. Mech., 43 (1976), pp. 198-202.

[34] B. B. ÖZHAN AND M. PAKDEMIRLI, Principal parametric resonances of a general continuous system with cubic nonlinearities, Appl. Math. Comput., 219 (2012), pp. 2412-2423.

[35] M. H. GHAYESH, S. KAZEMIRAD AND M. A. DARABI, A general solution procedure for vibrations of systems with cubic nonlinearities and nonlinear/time-dependent internal boundary conditions, J. Sound Vib., 330 (2011), pp. 5382-5400.

[36] K. V. AVRAMOV, Nonlinear modes of parametric vibrations and their applications to beams dynamics, J. Sound Vib., 322 (2009), pp. 476-489.

[37] N. S. NAmACHCHIVAYA, Non-linear dynamics of supported pipe conveying pulsating fluid. 1. Subharmonic resonance, Int. J. Nonlinear Mech., 24 (1989), pp. 185-196.

[38] N. S. NAMACHChIVAYA AND W. M. TIEN, Bifurcation behavior of nonlinear pipes conveying pulsating flow, J. Fluid Struct., 3 (1989), pp. 609-629. 